ÉTUDES

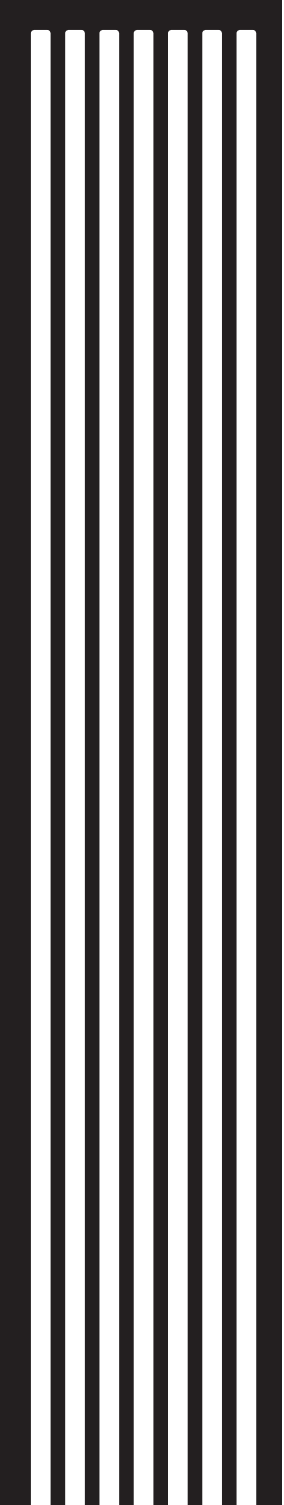





\title{
L'espace dans Un mort tout neuf d'Eugène Dabit, un cadre pour une écriture non engagée
}

\section{The Space in Un mort tout neuf by Eugène Dabit, a Framework for a not Engaged Writing}

\author{
Azucena Macho VArgas [azumava@unizar.es] \\ Universidad de Zaragoza, Espagne
}

\section{RÉSUMÉ}

Pour beaucoup d'auteurs, Eugène Dabit est uniquement un représentant de la littérature populiste. Il s'agit néanmoins d'un classement qu'il a toujours rejeté, considérant que ses œuvres ne répondaient pas aux postulats du mouvement. Toutefois, pour certains, Dabit était la voix du peuple car il parlait des classes moins favorisées à partir de sa propre expérience. Létude du roman Un mort tout neuf permet de montrer l'auteur sous un angle différent. En effet, la présentation d'un mode de vie et du cadre dans lequel il se déroule ont plus de poids que toute considération idéologique.

\section{MOTS-CLÉS}

Eugène Dabit ; littérature populiste ; espace ; description

\begin{abstract}
For many authors, Eugène Dabit is only a representative figure of literary populism. It is nevertheless a classification which he always rejected, considering that his works did not correspond to the postulates of the movement. However, for some, Dabit was the voice of the people because he spoke of the less privileged classes from his own experience. The study of the novel Un mort tout neuf allows to show the author in a different light. Indeed, the presentation of a way of life and the setting in which it takes place are more important than political ideology.
\end{abstract}

\section{KEYWORDS}

Eugène Dabit; literary populism; space; description

REÇU 2018-12-17 ; ACCEPTE 2019-02-25 
La trajectoire littéraire d'Eugène Dabit a été tronquée par une mort prématurée qui a aussi contribué à fixer une image peu réaliste de cet auteur. En effet, l'histoire littéraire s'occupe à peine de lui, le classant sous l'étiquette de populiste, envisageant plutôt ses amitiés que son œuvre romanesque. Ainsi, les questions politiques l'emportent sur les littéraires, permettant qu'un classement hâtif résume son œuvre.

L'objectif de cet article est double. D’une part nous tenterons de voir comment l'étude de l'œuvre de Dabit a été médiatisée par ses origines et la réception de ses romans et par une vision ultérieure chargée de préjugés. D’autre part, nous analyserons les éléments récurrents de la production dabitienne qui séloignent de cette image, en particulier ceux qui concernent une représentation de l'espace. En effet, le cadre de ses romans, (quartiers parisiens, cafés et habitations populaires), et les rapports entre celui-ci et les personnages qui l'occupent ouvrent la possibilité d’offrir une lecture différente, éloignée de l'engagement politique. Pour ce faire, l'axe central de cet article sera une œuvre peu connue, comme le sont, par ailleurs, la plupart des œuvres de cet auteur à l'exception d'Hôtel du Nord: Un mort tout neuf ${ }^{1}$, publié en 1934.

\section{Dabit écrivain populiste?}

Thibaudet ${ }^{2}$ affirmait qu'il serait impossible d'examiner de manière objective toute littérature contemporaine puisque la proximité empêcherait d'y porter un regard lucide. Se pencher sur ce qu'il appelle « littérature non-triée » peut en effet donner lieu à des classements hâtifs et l'histoire et la critique littéraire débordent d'exemples d'auteurs négligés car leurs contemporains les ont classés sous des étiquettes trop simplificatrices voire erronées. Le cas de Dabit nous en fournit un exemple intéressant : une vision intéressée de la part des critiques alliée à d'autres facteurs (sa mort prématurée et même le succès de l'adaptation d'Hôtel $d u$ Nord) ont contribué à cacher l'auteur et son œuvre sous le personnage.

L’entrée en littérature de cet auteur autodidacte, qui avait aussi tenté de vivre de la peinture, se fait en grande partie grâce au soutien de deux auteurs consacrés : Gide et Martin du Gard. C'est André Gide qui, le premier, lui trouve des qualités et l'encourage à continuer, puis ses dialogues avec Martin du Gard vont l'aider à trouver sa voie dans la littérature 3 .

Hôtel du Nord (1929) est couronné par le prix du Roman populiste et pour Dabit son acceptation implique aussi d’être considéré comme un romancier populiste, même s'il ne semble pas partager cette vision :

Ils veulent que je sois un romancier populiste... Il faut toujours qu'ils vous classent ! Qu'ils vous mettent dans le dos une étiquette ; ça leur sert peut-être à se tranquilliser [...] Quant

$1 \quad$ Nos références renvoient l’édition du roman chez Sillage (Dabit 2009)

2 «Il estimait qu'un critique [...] ne donne sa mesure que sur les auteurs anciens, classés, classiques et il se sentait embarrassé devant la production contemporaine qu'il qualifiait de « littérature non triée » (Leymarie 2006 : 153).

3 Gide mit en contact Dabit avec Martin du Gard, estimant qu'il « était mieux qualifié que [lui] pour orienter et qualifier une personnalité proche de la sienne "; si Dabit lui-même reconnaissait l'aide inestimable que Martin du Gard avait pour lui, Gide va jusqu’à affirmer que Hôtel du Nord, n’aurait jamais vu le jour « sans le conseil et l'appui constant de Martin du Gard, sans les encouragements de Chauveau » (Gide 1949). 
à l'histoire de ce "prix Populiste », ce que j’écrirai me permettra, j’espère, de me débarrasser de cette étiquette ${ }^{4}$.

Une mort prématurée l'empêcha de s'en débarrasser, toutefois cette affirmation est intéressante car elle montre à quel point le terme forgé par des théoriciens de la littérature aux origines bourgeoises éveillait les soupçons d'un auteur vraiment sorti du peuple. Le concept de "littérature populiste » faisait à l'époque l'objet d'interprétations diverses, car la « question de la représentation du peuple n'engage plus seulement des conceptions de la littérature, mais se discute sur le terrain social, politique et idéologique» (Paveau $1998: 49)^{5}$. Toutefois pour ses théoriciens, le populisme voulait surtout dénoncer la peinture du peuple qui apparaissait dans la littérature bourgeoise. En effet, elle reproduisait des clichés du XIX siècle, étant donné que les romanciers « éprouvaient de la difficulté à définir la place du prolétaire dans l'espace social et à circonscrire le cadre pour le mettre en scène" (Ouellet et Trottier 2013 : 8). Curieusement, cette prise de position a provoqué des critiques non seulement littéraires mais aussi politiques, venant de la droite mais aussi de la gauche. En effet, très souvent ces écrivains populistes sont moins engagés qu'ils ne le croient, n’oublions pas que ce sont des auteurs bourgeois pour lesquels le « peuple » n'est qu'un sujet exotique car ils « écrivent sur le peuple mais pas pour le peuple» (Tonnet-Lacroix 1993 : 30).

Dans le cas du roman primé de Dabit, et de manière générale de l'ensemble de son ouvre, une conception large du populisme est présente dans la mesure où le peuple lui-même fait l'objet d'une représentation littéraire. Mais aucune interprétation du terme ne correspondait à ce qu'il voulait transmettre dans ses romans. Ainsi, une réflexion sur l'image d'auteur, ou plutôt les images, s'impose, d'autant plus que « [...] en-dehors de toute question d'évaluation, il importe effectivement de les distinguer dans la mesure où les images de soi projetées par lécrivain ne sont pas du même ordre que les représentations de sa personne élaborées par des tiers » (Amossy 2009).

Cette image d'écrivain populiste contre laquelle Eugène Dabit avait l'intention de se battre vient d'abord du prix et s'explique par la thématique de toutes ses œuvres, dans lesquelles il insère des éléments autobiographiques. De plus, cette image semble se confirmer par son engagement politique et, pour certains, serait renforcée par son origine. Ainsi, pour Paul Nizan ses textes auraient plus de force que ceux d'autres auteurs, affirmant qu'il parlait « au nom des ouvriers » (Nizan 1936), même si Dabit lui-même ne sétait jamais présenté comme le porte-drapeau d'aucun mouvement, montrant que ce qui perdure est une représentation élaborée par ses contemporains.

Toutefois, il faudrait laisser de côté cette image d'auteur politisé, qui séloigne de celle qu'il voulait projeter pour faire une lecture de son œuvre dépourvue de tout parti pris politique. Gide était déjà partisan d'une approche différente et dans ses Feuillets d'automne il exprime aussi ses réticences sur le fait que l'on considère Dabit uniquement comme a working class novelist. De même il considérait que Villa Oasis renfermait une signification plus profonde qu'Hôtel $\mathrm{du}$ Nord mais il était convaincu que " par son sujet même, [le roman] est de nature à gêner certains partisans " (Gide 1949). Un point de vue qui s’opposait à celui d’auteurs comme Nizan qui considérait Villa

$4 \quad$ Lettre à R. Martin du Gard, 23 mai 1931.

5 Aucune péjorative, dans le sens où il est employé de nos jours. 
Oasis et Un mort tout neuf comme des œuvres de dénonciation ${ }^{6}$. Toutefois, Nizan avait critiqué Dabit, car il avait aussi publié des romans indignes d'un écrivain prolétarien. Ainsi, à propos de Petit Louis, publié en 1930, il affirme que c'est

le modèle des livres à condamner » parce que « M. Dabit décrit ainsi les ouvriers, comme du dehors, avec calme, avec désintéressement, avec toute la gratuité convenable aux romans de la maison Gallimard ». On y voit des ouvriers « vertueux et tranquilles », qui ne sont ouvriers « que par leur revenu qui est mince ». Eugène Dabit « offre donc des tableaux agréables aux bourgeois désœuvrés un peu fatigués des sauvages et des exotismes mondiaux». (Martinet $2011: 115$ ).

Ceci prouve que la réception de ces romans considérés populistes suscite les critiques de tous. Les auteurs considérés de gauche estimaient que les ouvrages étaient trop tièdes, offrant une image trop aimable des ouvriers, et reprochaient à l'auteur d'abandonner la lutte politique au profit de la littérature. De l'autre côté du spectre politique, on y voyait cependant des œuvres de dénonciation. Dans le cas d'Un mort tout neuf, au moment de sa parution, la plupart des critiques ont voulu y voir la confirmation de la veine populiste de Dabit. Il s'agit toujours d’approches qui privilégient une lecture idéologique, soit montrant un certain mépris envers ces gens auxquels « à peu près tout [...] manque pour être des âmes » (Charpentier 1934), soit le considérant un auteur engagé dans la défense des ouvriers uniquement parce qu'il retrace leur existence. C’est donc l'approche que l'on fait de l'œuvre plutôt que l'œuvre elle-même qui est à la base de la lecture politique.

\section{Présentation de l'espace urbain}

Ainsi, si nous suivons le conseil de Gide et allons au-delà de cette lecture idéologique, Un mort tout neuf nous permet aussi d’aborder l'étude de la représentation de l'espace dans l'œuvre de Dabit. D’une manière générale, les romans de Dabit privilégient la description : par exemple, dans Hôtel $d u$ Nord il cherche d'avantage à créer des ambiances et à dresser des tableaux quà faire le récit d'actions, comme le signale Nizan lui-même. Pour la même raison, l'espace chez Dabit est rarement un cadre vide, dans la mesure où il accueille les personnages :

À l’angle de la rue de Romainville et de la rue de Belleville, se trouve le Tabac. La Julie y entre. Au comptoir, ici, même les jours qui ne sont pas jours de marché, il y a du monde : le mécano qui a son petit atelier sur la place, le marchand de couleurs dont la boutique bariolée touche au mur du cimetière et le cordonnier, et le boucher, et le coiffeur (30-31).

Le premier élément qui écarte les romans d'un populisme déterministe est sans doute l'éloignement de l'esthétique naturaliste ${ }^{7}$. Il s'agit d'une manière de raconter et de décrire qui ne juge

6 Chez Nizan on perçoit même un certain reproche, comme s'il considérait que la littérature avait éloigné Dabit de l'engagement politique : "Dabit deepest urges push him towards the party of revolution, but he is held back in a vague literary world by umpteen bonds and formal scruples » (Redfern 1972:48).

7 Rappelons néanmoins que selon Paveau (1998: 54) «la matrice naturaliste de représentation du peuple» dans Hôtel du Nord est l'un des critères de la remise du Prix du Roman populiste. 
pas, et qui en principe manque de prétentions encyclopédiques ou idéologiques. Toutefois il est important de rappeler comme le signale Ziethen que « la production de l'espace fictionnel ne repose cependant pas uniquement sur ces informations textuelles mais sopère par les interférences d'un lecteur-modèle (Ziethen 2013 : 15). C’est pourquoi, l'espace décrit, ayant un référent réel qui nourrit l'illusion réaliste, devient un espace fictionnel auquel il est possible d'attribuer des valeurs axiologiques (voire politiques, comme nous l'avons signalé ci-dessus) en fonction du type de lecteur qui se penche sur le texte.

La représentation de l'espace dans le roman découle de mécanismes qui finissent par transformer un cadre neutre et souvent reconnaissable pour le lecteur en une construction qui va au-delà de sa présence physique, sur la page et dans la réalité qu’elle évoque (illusion réaliste), pour devenir une image véhiculant un contenu culturel souvent symbolique ${ }^{8}$. Ainsi, le Paris populaire des années trente, dépeint par Dabit, dépasse le statut de référent pour devenir un élément de la fiction. En effet, la description n'apparaît pas dans le récit au service du pittoresque, montrant la vie minable des petites gens qui s'acharnent au travail, tentant d'améliorer leur situation sociale. $\mathrm{Au}$ contraire, il se produit une identification entre l'espace et les personnages qui l'occupent qui se fonde sur le fait que ces descriptions de type voir (Hamon 1993) le regard dont le narrateur se sert pour décrire n'est que rarement celui d'un inconnu :

Elle l'aime 9 , leur coin. Presque en face de leur maison, c'est la rue du Télégraphe, la plus haute de Paris, dit-on, avec le vieux cimetière et les réservoirs de la Ville ; elle est large comme une place, provinciale, et deux fois la semaine s'y installe le marché. La rue de Belleville continue jusquà la porte des Lilas où l’on construit des immeubles énormes (22).

Cette identification, doublée d'un sentiment d'appartenance (partagé sans doute par Dabit luimême), favorise une présentation des cadres qui, sans séloigner de l'objectivité, investit l'espace d'une valeur axiologique. De ce fait, le plus souvent il s'agirait de descriptions représentatives (Adam, Petitjean, et Revaz 1989) ${ }^{10}$ qui, du fait du regard impliqué que l’on pose sur l'espace, enferment aussi une valeur expressive. Il s'agit le plus souvent de descriptions sans volonté encyclopédique ou systématique, les topographies se construisent à partir de touches insistant uniquement sur des détails pour construire un tableau ${ }^{11}$. Le cadre spatial est dépeint comme un espace vécu puisque ce sont les petits gestes ou les sentiments des personnages qui lui accordent sa véritable valeur. C'est le cadre anodin des quartiers populaires d'une ville, composé par des habitations modestes d'ouvriers, des cafés et des petites boutiques que Dabit réussit à transformer, créant une image mythique d'une ville liée à un mode de vie qui, tout en étant contemporain à l'écriture, dégage un sentiment de nostalgie et d'amour. Le regard est posé sur le cadre actuel mais la tête tient surtout compte du passé, car la mort et le temps transforment ce qui était présent en révolu. Ainsi, les yeux de Lucienne regardent « leur coin » qu’elle aime, parce qu'elle s'y sent à sa place,

8 Figuerola Cabrol (2008 : 78) signale comme preuve de l'importance du cadre dans les romans de Dabit le fait que la plupart d’entre eux y font appel dès le titre : villa, hôtel, banlieue etc.

$9 \quad$ Nous soulignons.

10 Rappelons que selon ces auteurs la description représentative aurait trois fonctions : la fonction mathésique, la fonction mimésique et la fonction sémiosique.

11 C’est un style pictural, qui rappelle que l'auteur était aussi un peintre de talent. 
sans pouvoir éviter d'éprouver un sentiment de nostalgie lorsqu'elle voit le changement d'une rue représentant un monde qui fera toujours partie de ses souvenirs. Le plus souvent dans le roman, la nostalgie révèle un lien évident avec le sentiment de perte car l'espace renferme des souvenirs qu'Albert connaissait aussi : "À bonne allure, le convoi descend la rue de Belleville. Hommes et femmes qui le composent connaissent cette rue - le mort aussi la connaissait !» (173).

Le même ton s'apprécie aussi dans la présentation de la petite maison de banlieue où habite la vieille Martha, la sœur ainée d'Albert Singer. C’est un petit pavillon auquel elle a rattaché une pièce pour avoir son indépendance. Néanmoins, malgré la taille exiguë du logement, on perçoit la fierté des gens qui ont réussi au prix de grands efforts, à avoir un chez soi indépendant.

L'après-midi, la vieille Marthe pousse près de la fenêtre son fauteuil et elle n'en bouge plus jusqu'au crépuscule. Elle tricote; ou approche son visage de la vitre, alors elle voit le jardin entouré d'un grillage rouillé. Lété, c'est sous le cerisier qu'elle passe ses après-midi ; l'hiver, elle en regarde les branches noirâtres derrière lesquelles apparaissent dans la rue de rares promeneurs. Quelquefois, elle lève la tête vers le ciel gris que traverse un avion venant du Bourget.

Le lotissement où elle vit avec son fils Michel, sa bru, et son petit-fils, s'appelle Adamville. Il date d'avant-guerre, aussi on y trouve des chemins pavés de mâchefer, des trottoirs de terre battue. Leur pavillon de briques se compose de deux pièces, pas plus ! son fils l’a payé quatorze mille - il n’a pas fini de s'acquitter de sa dette. Et elle, après la mort de son mari, avec ses économies, elle a fait construire une troisième pièce, sa chambre, dont elle ne sort guère. Elle ne se rend à Paris que pour voir son fils aîné : Adolphe, ou sa sœur Lucienne chez qui, parfois, elle rencontre ses frères. (105-106).

Le narrateur présente des quartiers populaires, sillonnés par des personnages qui y mènent une existence calme, circonscrite à l'arrondissement où ils ont leurs habitudes, mais le regard qu'il porte sur eux est cependant moins revendicatif que nostalgique. Épiceries, bars, tabacs... sont des endroits où les habitants se sentent à l'abri, se retrouvent et se reconnaissent. Ainsi, la nouvelle de la mort d'Albert Singer se répand rapidement dans le petit univers bellevillois car c'est le frère de la propriétaire du bar du Télégraphe et il y retournait souvent. Lucienne elle-même insiste sur le fait que ses frères, quoique profitant d'une bonne situation économique, ne renient pas de leurs origines et retournent à Belleville. Il se dégage de ces rapports un sentiment de fraternité et d’appartenance, car les gens s'y sentent à leur place et ressentent la solidarité des autres et aussi la compassion en raison de la perte subie.

La même situation se reproduit lorsque la nouvelle de la mort d'Albert arrive dans son quartier. Les rues qu'il fréquentait, les cafés où il avait ses habitudes, accueillent un monde quelque peu différent des habitants de Belleville, car ils jouissent d'une situation plus aisée, mais ils partagent le même sentiment d'incrédulité et de perte.

Côte à côte, ils remontent l'avenue. Ils sont grands, puissants, importants, et tiennent une bonne largeur du trottoir. Aux devantures étincellent des lumières ; des marchandes des quatre-saisons, des camelots, sont installés sur la chaussée. Au milieu de la cohue, des femmes font la retape, Gorin et le gros Édouard aperçoivent la môme Ida et sa copine Yvette, mais ils ne les saluent pas. Ils se répètent que par un soir comme celui-ci, mouvementé, gai, Albert était entre eux deux ; ou qu'ils allaient bientôt le retrouver dans sa boutique. La voici! Ils y entrent, leur chapeau à la main, la mine 
grave, et le gros Édouard est presque sans voix... À côté de la chemiserie, un bar où Albert prenait chaque matin son petit déjeuner. La nouvelle y arrive comme un coup de tonnerre, oui, la patronne en essuie une larme, et il faut accepter la tournée qu'elle vous offre. Cent mètres plus loin, son restaurant; puis un café tranquille, il y traitait des affaires de Bourse. (108)

C’est ainsi que se construit le sentiment de nostalgie : le décès d'Albert fait de cet univers bellevillois un monde révolu, qui n'existe plus car l'absence a changé l'état d'esprit des gens et même si le cadre où ils évoluaient est le même, les rapports varient puisque l'absence les a modifiés. Lorsqu'Edouard Singer, le frère ainé du décédé, va voir la voiture d’Albert ce sentiment est aussi mis en évidence ; le véhicule est présenté d’abord comme un symbole de la réussite économique d'Albert, mais c'est aussi l'image de la perte :

Mais, lorsqu'il en a ouvert la porte, le gros Édouard retrouve son frangin, avec ses manies, ses habitudes. Là, dans cette pochette, un plumeau, une paire de gants, des chiffons de laine ; ailleurs, un jeu de cartes de la région parisienne, une carte de la Côte d’Azur - le pauvre a eu raison de se payer ce beau voyage avant de mourir ; (160)

Ce regard empreint de tendresse et de nostalgie change lorsqu'il se pose sur le cadre où Albert et Paula ont vécu leur liaison, vu que l'appartenance cède le passage à l'étrangeté, à l'impression de regarder un univers éloigné : même si la distance physique est d’à peine quelques kilomètres, on y décèle une distance sociale. Curieusement ce quartier bourgeois, en principe plus riche et plus beau, n’est pas présenté comme charmant ou accueillant :

Cette petite rue étroite, bordée de villas laides et bourgeoises, derrière son père Gaston Dieulet s'y aventure. Dans cette maison triste, avec une grille où grimpe du lierre, Albert obscurément est venu mourir $(45)^{12}$.

Pour Gaston, le quartier semble sans âme et pas à cause de son aspect physique mais parce qu'il y manque de repères. Ceci s'avère vrai aussi lorsqu'il nous parle de l'intérieur de Paula, qui lui semble hostile alors qu'il aurait dû être considéré comme un exemple de richesse. Ce qui l'emporte est son impression que ce qui l'entoure ne constitue pas un véritable foyer, ce sont des objets mis en valeur dans le but d'être admirés, et le jeune homme se dit que son oncle, Albert Singer, enfant de Belleville comme lui, aurait sans doute éprouvé le même sentiment d'étrangeté.

Gaston regarde autour de lui. Paula aime les jolies choses. Sa villa est pleine de bibelots anciens ou modernes, d’objets d'ivoire et d'étranges fétiches nègres. Sur les murs sont accrochés des gravures, deux tableaux à sujet religieux, des peaux de serpent, un tapis de Perse, des dentelles. Albert, lorsqu'il est venu pour la première fois dans cette maison, il a dû s'imaginer pénétrer dans un musée $(63-4)$.

12 L'incongru de l'espace qui accueille le défunt est aussi souligné par son frère Edouard Singer : « D’un air sombre, le gros Edouard regarde autour de lui : des dentelles des fouillis, des gravures. On se croirait où ? Il sort de la chambre en marmonnant» (96). 
Il ne perçoit pas la maison comme un foyer où vivre, mais comme un musée. La propriétaire est néanmoins très fière de sa villa et elle affirme qu'Albert avait pensé pouvoir y vivre heureux : «Lorsqu'il a visité mon joli petit intérieur, continue Paula, il m’a confié : « Je sens que je m’y plairai » (51).

Le sentiment d'appartenance demeure donc déterminant dans les rapports entre espaces et personnages, et marque leur perception en tant que cadres d'accueil mais aussi en tant que modèles de beauté. Ainsi, si Paula est fière de son intérieur qu’elle considère de bon goût, Gaston demeure insensible au charme des lieux puisqu'il ne voit qu'un endroit étrange et rempli d’objets bizarres qu'il ne saurait considérer comme un espace accueillant.

Selon Figuerola Cabrol (2008 : 82-3), chez Dabit la présentation du cadre et le discours sur les rues parisiennes se redouble d'un code modal, explicite ou implicite, dans le sens où cette classe ouvrière décrite par le romancier « peut ou ne peut pas » habiter une partie ou l'autre de la capitale [...] la rue et, par analogie, le quartier sont devenus instruments d'une observation systématique de l'ensemble citoyen.

Ainsi, cette association personnages/espace demeure plus un constat de la distribution géographique des groupes sociaux dans la ville qu'un appel à la révolte. C'est un regard de sociologue tentant de peindre les mœurs d'un groupe social, lors d'une période précise et dans un espace précis, et le résultat tient plus des rapports modaux que de la prise de conscience politique.

\section{Espaces de la mort}

La peinture du cadre est très liée à ceux qui l’occupent, et pour cette raison il y a dans le roman des espaces qui renvoient à la mort, dont la maison de Paula. Pour la famille Singer, cette villa est un cadre hostile, espace de la mort et en quelque sorte responsable de la douleur provoquée, car à la disparition s'ajoute le fait quelle ne se soit pas produite dans un cadre familier.

De ce fait, même Paula va percevoir son propre foyer autrement : ce n'est plus un espace protecteur, mais une maison différente où elle ne se sent plus chez elle car la mort est devenue la maîtresse des lieux : «On ne sent plus d’odeur ; mais le silence pèse. Bref, elle se trouve dans sa villa comme une étrangère » (120). Elle se demande même si elle réussira à se débarrasser un jour de l'image d'Albert : « Elle changera le lit de place, achètera une couverture neuve. Peut-on chasser d'une maison l'image d'un mort? » $(100)^{13}$

Ce pouvoir de la mort s'appropriant des lieux avait déjà été traité par Dabit dans Villa Oasis. Lorsque la jeune fille poitrinaire décède, sa mère est incapable de rester dans un appartement où la défunte demeure omniprésente. La mort investit les lieux au point de bouleverser les rapports qui les lient à leurs occupants. Paula veut que le corps d'Albert disparaisse au plus vite pour pouvoir gommer ses traces, car c'est seulement de cette manière qu'elle pourra se sentir à l'aise chez elle. Ainsi, la douleur de la perte demeure secondaire, le choc initial passé, la propriétaire de la maison veut s'approprier à nouveau de son foyer. Tout en étant chez elle, elle partage le sentiment d'étran-

13 Face aux Singer, elle est consciente qu'elle ne pourra pas compenser les mauvais souvenirs de la mort par d'autres, car elle n’a rien partagé avec le mort : « Il ne lui reste de leur amour - ce nétait pas encore de l'amour - que des larmes, et de l'ennui ; et, dans sa jolie maison où personne nétait mort, cette puanteur qu'elle respirera pendant des semaines. Elle renverse la tête. Le désespoir l'envahit ; la colère et la haine s'y mêlent » (101). 
geté de Gaston Dieulet. Pour celui-ci, il y a quelque chose d'absurde dans le fait de veiller son oncle dans la maison d'une inconnue, sans avoir rien à se dire car ils n'ont aucun souvenir commun du mort $^{14}$. De plus, la nuit investit l'espace et tout est perçu autrement :

La lueur des deux appliques éclaire le visage du mort. Lombre envahit les murs. Dans la glace, glauques et figés, se reflètent le lit, le cadavre, les formes étranges de quelques meubles. Deux tableaux, images privées de mouvement, choses mortes ; des vases, sur une commode ; tentures lourdes, aux longs plis mortuaires; fauteuils où la place d'un corps... [...]. Dans l'air immobile, une odeur légère, puis envahissante, elle monte du mort qui n’a pas rompu tous liens avec son passé, de son corps où d'incertaines transformations commencent. Qu'elle s'étale et emplisse le vide, quelle pénètre le bois, les étoffes, la pierre, pour les tirer de leur monde inanimé. (82)

Létrangeté est partagée par Edouard Singer et Ferdinand Dieulet, lorsqu'ils visitent avec Gaston l'appartement du défunt pour chercher ses papiers. L'impression de vide et d'absence vont de pair avec le sentiment dêtre des intrus dans un espace autrefois familier mais transformé par la mort.

C’est un appartement de deux pièces, intime, propre ; seulement, quatre personnes y sont à l'étroit. Ils piétinent dans la salle à manger, en lorgnant à droite, à gauche, avec curiosité, et aussi une espèce d’inquiétude. Il leur semble que le frangin va se présenter, leur demander : «Vous faites quoi ? Vous êtes-vous essuyé les pieds, au moins?» (126)

En réalité, l'espace est inchangé, mais ce « mort tout neuf » perturbe leur regard car ce cadre qui était lié à quelqu'un semble montrer à peine les traces de celui qui l’a occupé, disparaissant dans le brouillard. («Où se posent leurs regards ils voient des choses poussiéreuses, tristes, immobiles, comme si la mort les avait frappées » 139) De plus, parmi les documents trouvés, le narrateur s’attarde en particulier sur les petites annonces publiées et les lettres échangées avec ces inconnues, qui offrent à la famille la face cachée du défunt. La douloureuse prise de conscience qu'ils n'avaient de lui qu'une connaissance partielle vient augmenter leur malaise.

Si les cadres se définissent le plus souvent par des rapports d'appropriation et appartenance, l'appartement d'Albert porte les traces de quelqu'un qui sera absent pour toujours, devenant en quelque sorte, un espace à réinvestir, puisqu'il sera occupé par d’autres. L’absence devient un élément transformateur de la vie mais aussi du cadre où elle se déroule. En effet, de la description se dégage un sentiment de nostalgie évoquant un temps apparemment révolu qui ne l'est que parce que la mort transforme la représentation et la perception des lieux, l’espace reste inchangé mais ce jour, celui où le mort est « tout neuf » les rapports espace-personnages changent. Ainsi, l'absence même d'un personnage devient un élément transformateur non qu'elle change vraiment l'espace mais le regard que l'on porte sur lui.

Et le vieil Ernest approche son visage de la glace. Il aperçoit un boulevard avec des arbres nus, des murs gris. « Le boulevard Ornano », lui crie Lucienne. La porte de Clignancourt est entourée

14 L'espace dans le récit va au-delà de la simple topographie pour sériger comme un élément à valeur pragmatique qui agit et fait agir les personnages. (Macho Vargas 2010 : 146) 
de hautes bâtisses. C'est froid, monotone, impitoyable. Il retombe dans son coin, au fond de son passé. (175)

Toutefois le regard empreint de nostalgie est présent dès le début, avant même l'annonce de la mort, lorsque Lucienne contemple son quartier et le décrit en se souvenant sans doute des moments de bonheurs vécus et se rend compte de la transformation qu'il a subie.

Le seul espace qui éprouve vraiment une transformation après la mort d'Albert Singer est le Bar du Télégraphe. Lucienne Dieulet, sa propriétaire, y reçoit son frère pour la dernière fois. Le petit bar populaire est devenu un monument au mort, car comme elle le dit, même une cérémonie civile peut « avoir de la tenue » : «Leur coin, si familier, si calme, le voici tout changé et solennel » (163). Le bar drapé de noir est transformé pour honorer le défunt, au point que Ferdinand « : quant à lui... hé, lui se demande si demain les clients entreront de bon cœur au Bar du Télégraphe ?» (163). Il est conscient que la perception du cadre est plus importante que le cadre lui-même, et il espère que chez ses clients l'image du bar comme un espace de mort disparaitra en même temps que le cercueil, que la vie de quartier reprendra et que ses habitués reviendront à son commerce.

Dabit reprend aussi dans ce roman une idée déjà traitée dans Villa Oasis : le caractère égalisateur de la mort qui finit par atteindre tout le monde, anéantissant les rêves. De même, il insiste sur l'importance que l'ultime demeure possède pour les personnages : l'ascension sociale doit aussi être mise en évidence après la mort, d’où l'importance de l'image du caveau comme symbole de réussite. Cela renforce l'affirmation que l'espace, chez Dabit, n'est jamais un cadre vide mais renferme un sens pour les personnages qui l'habitent et le parcourent : c'est l'expression de leur appartenance à un groupe social et résume leur existence. Il en est de même lorsque cet espace est le caveau, car pouvoir montrer au monde qu'il s'agit d'un espace marqué, renforce chez eux le rapport d'appartenance et nourrit leur fierté, étant donné qu'il s'agit du dernier lieu où pouvoir faire étalage de richesse.

Curieusement, tout comme dans Villa Oasis les rêves sont doublement détruits ; d'une part, la mort tronque les projets de mariage, de l'autre, le caveau d'Édouard qui doit accueillir Albert Singer, est mal construit et le cercueil y rentre à peine. Si Albert n'avait exprimé aucun souhait concernant ses funérailles ( Tu pourras me mettre où tu veux ; lorsque je serai mort, je serai bien mort » (87)), pour Edouard, ce caveau défectueux représente une trahison à son frère (même si celui-ci ne lui avait rien demandé) : "Une sensation horrible loppresse, une sorte de remords, comme s'il n'avait pas fait tout le nécessaire pour son vieux frangin et participé à ce sabotage » (179). De plus, le défaut de construction lui montre aussi que sa réussite peut se transformer en échec. L'importance du caveau à ses yeux dépasse celle de l’argent : symbole et demeure (« Je m’en fous à présent du pognon, je ferai reconstruire mon caveau » (180)).

La mort d'Albert et le fait de devoir affronter une perte inattendue marquent un tournant dans l'existence des Singer. Ce " mort tout neuf », comme le narrateur l'appelle, deviendra bientôt un mort vieux. Entre temps, il est devenu le symbole de la prise de conscience de l'écoulement du temps, de son pouvoir transformateur et du caractère éphémère de l'existence :

Avec Albert ! crie Ferdinand au vieil Ernest, quand on était gosses, on est venus souvent ici, à cause de la foire aux puces. Tu te rappelles? 
Aujourd'hui, pas d'étalages, peu de promeneurs. Les guinguettes sont fermées ; une seule est ouverte, il en monte une odeur de graisse et de vin aigre. Des hommes, français ou indigènes, manœuvres, vagabonds, chômeurs, traînent une existence dont ils ne savent que faire, près du cimetière qui les attend ; ou bien suivent cette avenue qui file vers Saint-Denis rejoindre d'autres avenues, puis des routes qui, elles, traversent des villages où poussent de vrais arbres, où les cimetières sont paisibles. (181)

Cette prise de conscience établit une frontière invisible qui sépare la famille Singer, dont le monde est bouleversé, et les habitants de la ville qui nont pas vécu le même bouleversement car pour eux le mort ne représente rien et, étrangers à leur perte, ils continuent à mener la même existence, dans les mêmes lieux : Personne ne sait qu'il y a un mort, ici ? et, dans Paris, qui pense qu'il y a un mort de plus? Trois janvier, lendemain de fête ; la vie active va reprendre (84).

\section{Conclusion}

Un mort tout neuf prend comme point de départ, comme d'autres romans de l'auteur, une anecdote autobiographique qu'il agrandit pour nous montrer un morceau de vie. Ceci s'avérait déjà dans Hôtel $d u$ nord et le fait de brosser la routine du microcosme qui évoluait autour de l'hôtel du quai Jemmappes a valu à l'auteur une étiquette avec laquelle il ne s'est jamais senti identifié. Si dans Hôtel du nord le véritable héros du roman et son "personnage " plus connu est l'hôtel, dans Un mort tout neuf ce n'est pas Albert Singer, mais la mort elle-même.

Si nous considérons le roman du point de vue de l'importance de l'espace, sa représentation et les rapports avec les personnages, ce roman échappe à létiquette du populisme à laquelle est réduite trop souvent l'œuvre de Dabit. Le texte nous offre une vision plus bienveillante que revendicative des quartiers populaires de Paris (bars au comptoir de zinc, rues vivantes et habitations humbles). Tout comme dans d'autres romans, Dabit brosse l'existence de gens qui travaillent dur, fiers de gagner leur vie et qui aspirent à vivre aisément, prenant comme argument la mort de l'un d'entre eux.

Par le biais des changements que la mort introduit dans le quotidien, changeant la perception des autres, dévoilant l'espace d'une manière différente, la présentation de l'espace construit des tableaux présidés par un double sentiment d'appartenance à un monde et de nostalgie d'une époque que la mort transforme en révolue.

\section{Références bibliographiques}

Adam, J.-M. ; Petitjean, A. ; \& Revaz, F. (1989). Le texte descriptif: poétique historique et linguistique textuelle, avec des travaux d'application et leurs corrigés, Nathan université. Paris : Nathan.

Amossy, R. (2009). La double nature de l'image d'auteur. Argumentation et Analyse du Discours, 3.

Charpentier, J. (1934). Les Romans. Un mort tout neuf. Mercure de France, 30 septembre 1934.

Dabit, E. (2009). Un mort tout neuf. Paris : Sillage. [Édition originale : NRF, 1934] 
Figuerola Cabrol, C. (2008). Lieux magiques ou maudits ? Autour du Paris d'Eugène Dabit. In S. Bernard Griffiths, \& A. Santa (Eds.), Lieux magiques. Magie des lieux (pp. 77-92). Clermont-Ferrand : Presses Universitaires Blaise Pascal.

Gide, A. (1949). Feuillets d’automne précédés de quelques récents écrits. Paris : Mercure de France.

Hamon, P. (1993). Du descriptif. Hachette université. Recherches littéraires. Paris : Hachette Livre.

Leymarie, M. (2006). Albert Thibaudet : «loutsider du dedans». Villeneuve d’Ascq : Presses universitaires du Septentrion.

Macho Vargas, A. (2010). À propos des espaces dans Villa Oasis d'Eugène Dabit. Roman 20-50, 50 (2), 137-146.

Martinet, J-L. (2011). La construction d’une figure auctoriale révolutionnaire : Paul Nizan (1929-1933). Interférences littéraire/Literaire interferenties, 6, 101-118. <http://www.interferenceslitteraires.be/index.php/ illi/article/view/591/462>

Nizan, P. (1936). L’ouvre d'Eugene Dabit. L'Humanité, 6 septembre 1936.

Ouellet, F. ; \& Trottier, V. (Eds.) (2013). Populisme pas mort. Autour du Manifeste du roman populiste (1930) de Léon Lemonnier. Études Litteraires, 44 (2) <https://www.erudit.org/fr/revues/etudlitt/2013-v44-n2etudlitt01269/>.

Paveau, M.-A. (1998). Le « roman populiste» : enjeux d'une étiquette littéraire. Mots. Les langages du politique, 55, 45-59.

Redfern, W. D. (1972). Paul Nizan: Committed Literature in a Conspiratorial World. Princeton University Press.

Tonnet-Lacroix, É. (1993). La littérature française de lentre-deux-guerres (1919-1939). Paris : Nathan. Collection Fac Littérature.

Ziethen, A. (2013). La littérature et l'espace. Arborescences. Revue d’études françaises, 3. doi: 10.7202/1017363ar. 